\title{
The topology of neuronal structures exposed to cosmic radiation
}

\author{
ARMEL MAGANGA MIHINDOU \\ Faculty of Sciences, Rabat \\ Department of Mathematics \\ 4 Avenue Ibn Battouta B.P. 1014 RP, Rabat \\ Rabat, MOROCCO
}

\author{
MY ISMAIL MAMOUNI \\ CRMEF Rabat, Morocco \\ Department of Mathematics \\ Av. Allal Al Fassi, Madinat Al Irfane \\ Rabat, MOROCCO
}

\begin{abstract}
In this paper, we focus on some leader NASA experiences to explore how cosmic radiation caused significant reductions in dendrite and spine complexity. We adopt a topological data analysis approach and extract more information then the classical methods. Our key idea is to use the NASA images of the neural networks of some mouses that were exposed 12 weeks to cosmic radiation. We associate to this neural network code bares that giveusmoreinformation, thatthatgivenbytheoriginalexperiences.
\end{abstract}

Key-Words: topological data analysis, persistent homology, cosmic radiation, image analysis, segmentation.

Received: October 28, 2019. Revised: May 6, 2020. Accepted: May 18, 2020. Published: May 28, 2020.

\section{Introduction}

Since human has gave himself the mission to conquer the space, and then inevitably be exposed to cosmic rays, the cosmic radiation became a hot topic that catches the attention of the medias, politicians, scientists, ... Due to the increased use of the technology supports (as smart phones), families are more and more interested in rays negative effects on their children, and are asking a lot of questions, like : How to protect efficiently the neural network of our navigators agents from cosmic ray? How to predict the impact of cosmic radiation on neural network

Cosmic rays are high energy radiation particles, mainly originating outside the Solar System. Composed primarily of high energy protons and atomic nuclei, they are of mysterious origin. The origin of the cosmic radiation is an old subject of discussion : E. Fermi (see [7]) proposed a theory according to which cosmic rays are originated and accelerated primarily in the interstellar space of the galaxy by collisions against moving magmetic fields. R. D. Richtmyer and E. Teller (see [15]) has advocated the viewpoint that the cosmic rays are of solar origin and are kept relatively near the sun by the action of magnetic fields.

NASA hopes to send the first round-trip, manned spaceflight to Mars by the 2030s. Studies in mice suggest that these particles could alter the shape of neurons, impairing astronauts memories and other cognitive abilities. Indeed, nervous system risks which include during space missions and lifetime risks due to space radiation exposure are of concern for long-term exploration missions to Mars or other destinations. Possible risks during a mission are altered cognitive function, including detriments in short-term memory, reduced motor function, and behavioral changes, which may affect performance and human health.

So, should we worry? The answer is yes : energies of the most energetic ultra high energy cosmic rays have been observed to approach $3 \times 10^{20} \mathrm{eV}$ and about 40 million times the energy of particles accelerated by the Large Hadron Collider. ${ }^{1}$ Thus and even after 54 years of sending astronauts into space, NASA is still learning about how space conditions affect the human body. The agency is studying the effects of long-duration space flight on human vision, with its unique twins study, and researchers continue to analyse how micro gravity wears away at the musculoskeletal system.

In this paper, we focus on some leader experiences (e.g. [12], [13], [14]), after what authors are convinced that irradiation caused significant reductions in dendrite complexity, spine density and altered spine morphology along medial prefrontal cortical neurons known to mediate neuro transmission interrogated by our behavioural tasks. Behavioural deficits for individual animals correlated significantly with reduced spine density and increased synaptic puncta, providing quantitative measures of risk for developing cognitive impairment.

Thanks to the persistence homology, as an algebraic topology tool used in the topological data analysis approach, we propose to issue a barcode of the

\footnotetext{
${ }^{1}$ The worlds largest and most powerful particle accelerator.
} 
results of these experiences. The only data we will use is high-resolution imaging of brain tissue taken by the authors for rodents' brain before and after having bombarded them with some cosmic radiation. We will implement an algorithm that store regions adjacency under topology signature to evaluate the density of dendrites and spines. Moreover, we will design a software which issue from any humain IRM's brain an alert about the neuronal density. This can be useful, for example, to alarm the parents whose kids are addict of video games, they only need brain scan to put in the software. An alert will be issued according to the dendrite complexity and spine density readed by the software, based only on brain scan. Indeed, persistent homology persistence allowed us to interpret geometrical constitution shape, and then can be used to make shape recognition.

The paper is organized as follows : In section 2 we will briefly describe the neural network and how cosmic rays can damage it. Section 3 is the theoretical part devoted to summarize the persistent homology concept, while in the section 4 we will present our ideas to analyse topologically some NASA's experiences on the possible negative impacts of the cosmic radiations on some behavior tasks. Our contribution is a shape recognition algorithm based on the persistent homology viewpoint that analyzes the geometry and the topology of the spines and dendrites distribution. Finally, in the section 5, we will present a software which can be used by doctors to predict the dendrite complexity and spine density based (for example) on a brain IREM image. This can be useful to alert parents whose children are addict to high resolution radiation video games.

\section{The Human brain and cosmic ra- diation}

The neural networks are a neural arrangement, each neural is extended by a dendrite topped with thorns called spines. These spines play an important role in the communication between neural.

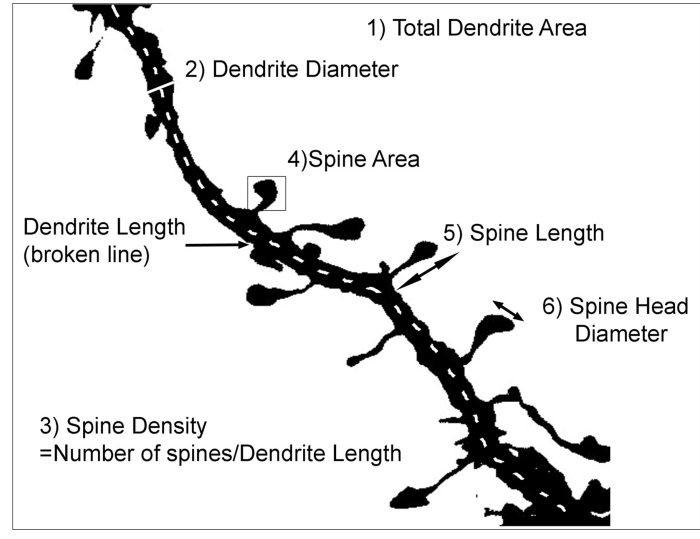

Figure 1: Structure of a typical dendrite

Dendrites are the branches of neurons that receive signals from other neurons and pass the signals into the cell body. The axon is the part of a neuron that sends the signal. Axons that seel like a highly developed dendrite are long and thin. The axon carries an electrical signal from the cell body to the synapse : the structure that permits a neuron to pass an electrical or chemical signal to another neuron. Synapses are essential to neuronal function: neurons are cells that are specialized to pass signals to individual target cells, and synapses are the means by which they do so : A dendrite from one neuron and an axon from another neuron meet at a synapse.

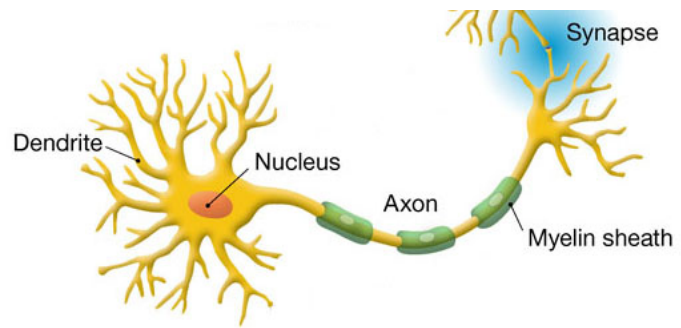

Figure 2: Communication process between two neurons

The cosmic radiation is a phenomena caused by the unstable atom kernels that are spread in the space, it is unavoidable that these displacements are not without risk especially for human being, since they can affect the neural network. The well known Ch. L. Limoli team have to make an important breakthrough : to select the best candidates for a one-way trip to Mars. For this purpose, the molecular biologist at the University of California, Irvine, and his colleagues took male mice to a particle accelerator at the NASA Space Radiation Laboratory in Upton, New York. There, they catapulted oxygen and titanium ions down a 100meter transport tunnel and into the restrained rodents's brains at roughly two-thirds the speed of light.

Six weeks after exposure, researchers at UC Irvine noticed distinct changes to the brains of the 
mice notably inflammation that disrupted communication between the neurons. According to the study, the particles acted like tiny bullets, flying into the brain and breaking off neuronal structures known as dendrites. The loss of these branch-like synapses, which carry electrochemical signals between neurons, are often associated with cognitive impairments and Alzheimers disease.

Going one step further, the researchers did some behavioral experiments with the exposed mice to see how these brain changes might affect their memory and learning abilities. Sure enough, the mice exhibited less curiosity and seemed more confused than mice who had not been exposed to space-like radiation. These symptoms are similar to the cognitive changes brain cancer patients experience when undergoing radiation treatments.

\section{Topological data analysis}

Topological Data Analysis (TDA) is an emerging trend in exploratory data analysis and data mining. It has known a growing interest and some notable successes (such as the identification of a new type of breast cancer, or the classification of NBA players, or the prediction of the future USA president) in the recent years. Indeed, with the explosion in the amount and variety of available data, identifying, extracting and exploiting their underlying structure has become a problem of fundamental importance. Many such data come in the form of point clouds, sitting in potentially high-dimensional spaces, yet concentrated around low-dimensional geometric structures that need to be uncovered.

The non-trivial topology of these structures is challenging for classical exploration techniques such as dimensionality reduction. The goal is therefore to develop novel methods that can reliably capture geometric or topological information (connectivity, loops, holes, curvature, etc) from the data without the need for an explicit mapping to lower-dimensional space. Persistent homology is the main tool of the TDA, it consists to represent any shape under a barcode. As the saying goes, "every data have a shape, and any shape have a meaning". Thus the key idea of TDA is to represent a data as a shape (a point cloud for example), and the issue its barcode (the meaning of the data).

In the TDA community we agree, without formalizing it, that $\mathrm{H}$. Edelsbrunner is the founder of this theory Indeed, the fast algorithm described in his leader paper [4] triggered the explosion of interest we currently observe because its availability as software facilitates the application to a broad collection of prob-

lems and datasets.

We agree also that the G. Carlsson research works (e.g., [2], [3], [17]), and that the software platform and applications offered by its machine intelligence software company Ayasdi, are the precursors of the current popularity of TDA both in the scientific and industrial communities. For example, one widely reported top five global systemically important bank was that to build models required for the annual Comprehensive Capital Analysis and Review (CCAR) process took 1,800 person-months with traditional manual big data analytic and machine learning tools, but took 6 person-months with Ayasdi. Now, Ayasdi, founded in 2008, is considered now as "A Big Data Start-Up With a Long History", and recently announced a new 55 million USD round of Series C funding, led by Kleiner Perkins Caufield \& Byers (KPCB), and joined by existing investors, Institutional Venture Partners (IVP), Khosla Ventures, FLOODGATE, Citi Ventures, and new investors, Centerview Capital Technology and Draper Nexus.

\subsection{Simplicial homology}

Simplicial homology refers to two concepts, that of abstract simplicies, which form a family of sets that is closed under deletion of elements, and that of geometric simplicies, which are geometric object of various dimensions (points, line segments, triangles, tetrahedra, and so on), glued together according to certain rules. As we will see in a moment, the two concepts are in fact closely related and can give some kind of information about the Euclidean space considered.

Definition 1. Given some fixed points $\left(e_{i}\right)_{0 \leq i \leq n}$ in $\mathbb{R}^{m}$, their associated $n$-simplex is the convex hull

$$
\left[e_{0}, \ldots, e_{n}\right]:=\left\{\sum_{i=0}^{n} t_{i} e_{i}, t_{i} \geq 0, \sum_{i=0}^{n} t_{i}=1\right\} .
$$

Hence, a 0 -simplex is a vertex, a 1-simplex is an edge, a 2-simplex is a full triangle, while a 3 -simplex is a full pyramide.
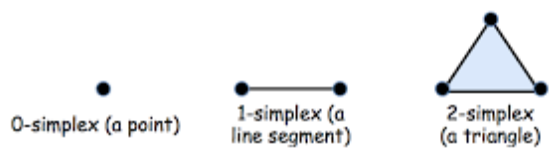

Figure 3: Examples of simplices

The convex hull of any non empty subset of this $n+1$ points define an simplex, called a face of the simplex.

\footnotetext{
${ }^{2}$ The New York Times. January 16, 2013.
} 
Note that faces are simplices themselves, and that the empty set is a face of every simplex.

Definition 2. A simplicial complex $\mathcal{K}$ is a set of simplices that satisfies the following conditions:

1. Every face of a simplex from $\mathcal{K}$ is also in $\mathcal{K}$;

2. The intersection of any two simplices $\sigma_{1}, \sigma_{2} \in \mathcal{K}$ is a face of both $\sigma_{1}$ and $\sigma_{2}$.

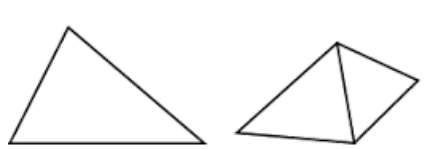

simplicial complexes

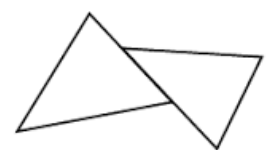

not a simplicial complex
Figure 4: Simplicial complex or not?

A $n$-chain in $\mathcal{K}$ with coefficients in $\mathbb{Z}_{2}$ is any formal sum $\sum n_{i} \sigma_{i}$, where $n_{i} \in\{0,1\}$ and $\sigma_{i}$ is a $n$ simplex in $\mathcal{K}$. The subset of all this $n$-chains will be denoted $\mathcal{C}_{n}(\mathcal{K})$, with the convention that $\mathcal{C}_{-1}(\mathcal{K})=\emptyset$.

Definition 3. The boundary operator is the $\mathbb{Z}_{2}$-linear map defined by :

$$
\begin{aligned}
\partial_{n}: \quad \mathcal{C}_{n}(\mathcal{K}) & \rightarrow \mathcal{C}_{n-1}(\mathcal{K}) \\
\sigma:=\left[e_{0}, \ldots, e_{n}\right] & \mapsto \partial_{n} \sigma
\end{aligned}
$$

where, $\partial_{n} \sigma:=\sum_{i=0}^{n}(-1)^{i}\left[e_{0}, \ldots, \hat{e}_{i}, \ldots, e_{n}\right]$ and that $\hat{e_{i}}$ means omitted.

For example $\partial_{0} A=0, \partial_{1}[A, B]=B-A$, while $\partial_{2}[A, B, C]=[B, C]-[A, C]+[A, B]$, which well justifies the appellation boundary. It is easy to show that

\section{Theorem 1.}

$$
\partial_{n-1} \circ \partial_{n}=0 .
$$

In particular we have

$$
\operatorname{Im} \partial_{n} \subset \operatorname{ker} \partial_{n-1} .
$$

This yields the chain complex

$0 \stackrel{i}{\hookrightarrow} C_{n}(\mathcal{K}) \stackrel{\partial_{n}}{\longrightarrow} C_{n-1}(\mathcal{K}) \stackrel{\partial_{n-1}}{\longrightarrow} \ldots \stackrel{\partial_{1}}{\longrightarrow} C_{0}(\mathcal{K}) \stackrel{\partial_{0}}{\longrightarrow} 0$,

which is an algebraic structure that consists of a sequence of abelian groups (or modules) and a sequence of homomorphisms between consecutive groups such that the image of each homomorphism is included in the kernel of the next. Elements of $\operatorname{Im} \partial_{k}$ are called boundaries, those of ker $\partial_{k-1}$ are called cycles. Thus any boundary is a cycle, the inverse is not always true.
Definition 4. The $k$-th simplicial homology group of $\mathcal{K}$, is defined to be the quotient group

$$
H_{k}(\mathcal{K})=\operatorname{ker} \partial_{k-1} / \operatorname{Im} \partial_{k} .
$$

Its rank, denoted $\beta_{p}(\mathcal{K})$, is called the $k$-th Betti number of $\mathcal{K}$.

$H_{k}(\mathcal{K})$ represents the obstruction of a cycle to be a boundary, and $\beta_{p}(\mathcal{K})$ represents the number of the homologus $k$-dimensional holes in a shape. Since the interior of a circle is a disc, which is a variety of dimension 1 , one may consider a circle to have a onedimensional hole. In particular $\beta_{0}$ is the number of the path-conected components of a shape, since two points are homotopic if and only if they live in the same path-conected component.
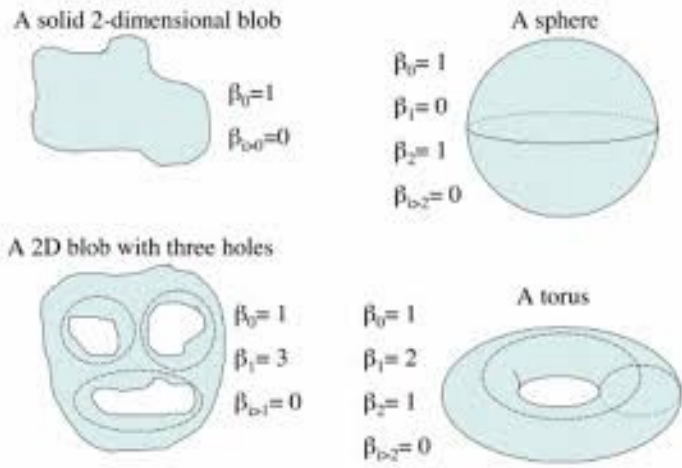

Figure 5: Betti numbers of some shapes

For further and deep details on the notion of homology, we recommand to the reader this standard references : [8] and [9].

\subsection{Persistent homology}

Persistent homology is a kind of a homology theory based on the notion of filtration.

Definition 5. Given a simplicial complex $\mathcal{K}$, a filtration of $\mathcal{K}$ is any nested sequence of sub-complexes defined by:

$$
\text { 1. } \emptyset=\mathcal{K}^{0} \subset \mathcal{K}^{1} \subset \cdots \subset \mathcal{K}^{n}=\mathcal{K} \text {; }
$$

2. $\mathcal{K}^{i+1}=\mathcal{K}^{i} \cup \sigma^{i+1}$, where $\sigma^{i+1}$ is a simplex of $\mathcal{K}$.

Let $\mathcal{C}_{k}^{p}$ denote the set of the $k$-chains (with coefficients in $\mathbb{Z}_{2}$ ) of $\mathcal{K}^{p}$, then the restriction of the boundary operator is $\partial: \mathcal{C}_{k}^{p} \longrightarrow \mathcal{C}_{k-1}^{p-1}$. Let $\mathbb{Z}_{k}^{p}$ and $B_{k}^{p}$ denote respectively the sets of $k$-cycles and $k$ boundaries of $\mathcal{K}^{p}$, then one may check obviously the following inclusions

$$
\begin{aligned}
& Z_{k}^{0} \subset Z_{k}^{1} \subset \cdots \subset Z_{k}^{p} \subset \ldots Z_{k}^{n}, \\
& B_{k}^{0} \subset B_{k}^{1} \subset \cdots \subset B_{k}^{p} \subset \ldots B_{k}^{n} .
\end{aligned}
$$


Definition 6. For some fixed $p \in\{0, \ldots, n\}, q \in$ $\{0, \ldots, n-p\}$, the $k$-th persistent homology group of $\mathcal{K}^{q}$, denoted $H_{k}^{q, p}$ is the quotient group defined by

$$
H_{k}^{q, p}:=Z_{k}^{p} /\left(B_{k}^{p+q} \cap Z_{k}^{p}\right)
$$

Its rank is called the $k$-th persistent Betti number of $\mathcal{K}^{q}$.

The $k$-th persistent Betti number of $\mathcal{K}^{q}$ represents the number of the independent homology classes of $k$ cycles in $\mathcal{K}^{q}$ that are not boundaries in $\mathcal{K}^{q+p}$. Intuitively, a $k$-cycle in $\mathcal{K}^{q}$ generating a non zero element in $H_{k}^{q, p}$ is a cycle that has appeared in the filtration before the step $q+1$ and that is still not a boundary at step $q+p$.

To understand how the topology of the filtration evolves each time we add a simplex, we say that the simplex $\sigma^{p}$ is positive if it is contained in a $(k+1)$ cycle in $\mathcal{K}^{p}$, which is necessarily not a boundary in $\mathcal{K}^{p}$ and negative otherwise. With the above definition the $k$ - th Betti number of $\mathcal{K}^{p}$ is equal to the difference between the number of positive $k$-simplices (which are creating $k$-cycles) and the number of negative $(k+1)$ simplices (which are killing $k$-cycles).

Thus, a homology class is created when a positive simplex is added in the filtration and that a homology class is destroyed when a negative simplex is added. Topological persistence provides a natural way to pair positive and negative simplices such that whenever a positive simplex is added to the filtration it creates a homology class and a corresponding cycle that becomes a boundary when its paired negative simplex is added.

A nice way to represent this pairing is to consider the couple $\left(\sigma^{j}, \sigma^{\ell(j)}\right)$, where $\sigma^{j}$ is a negative simplex and $\ell(j)$ the largest index of the positive $k$-simplices associated to $\partial \sigma^{j}$. Drawing this pairs, we get the persistent diagram, whose horizontal axis indicates the birth of a cycle, and whose vertical one indicates its death. The "life" of a cycle, which represents the persistent of a given data, is equal to the height of its associated pair computed from the diagonal (since the diagonal interpret the birth time of a cycle).

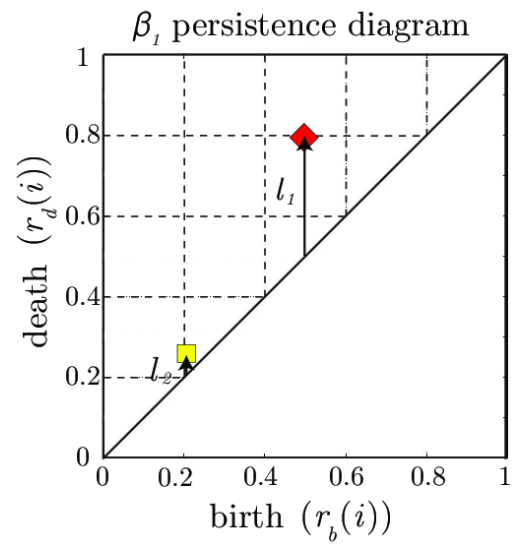

Figure 6: Example of a persistent diagram

Another equivalent way to represent this pairing birth-death is the barcode, which is a graphical representation of the evolution of the homology of a given simplicial complex following the chosen filtration. That is a collection of horizontal line segments in a plane whose horizontal axis corresponds to the lifetime (the persistence) of a homology generator and whose vertical axis represents an (arbitrary) ordering of homology generators.

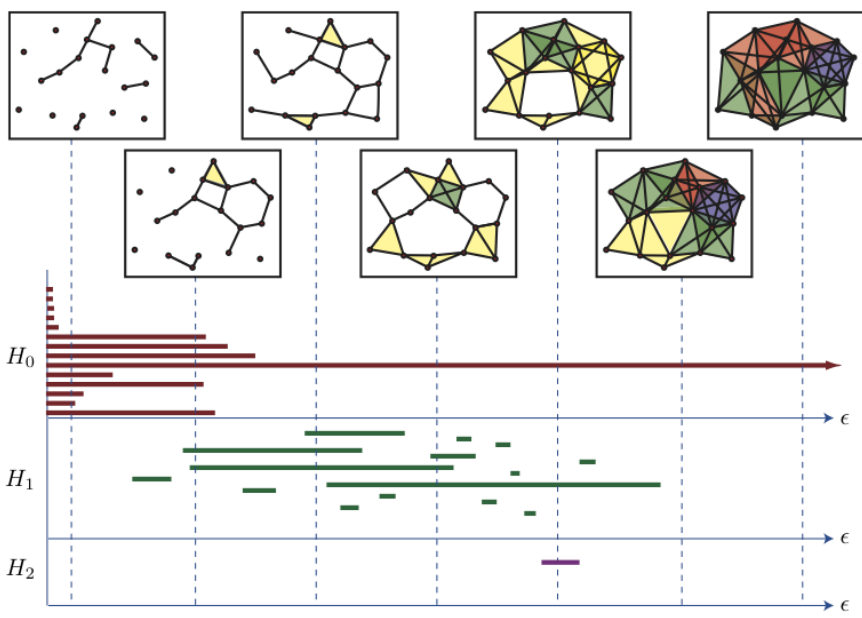

Figure 7: Example of a filtration, and its associated barcode

The attentive reader will certainly infer that for a fixed simplicial complex (a data represented as a point cloud), the barecode issued depend on the choosen filtration. There are several approach (VietorisRips, Cêsh, alpha, weak witnes, Delaunay, ...), and one have to choose the most adapted to the context of his problematic. For more and further details, we refer the interested reader to the successful comparison [11]. For more general information on persistent homology we advise this standard references : [5], [6]. 


\section{Topological data analysis of some NASA experiences}

Nervous system risks during space missions due to space radiation exposure, are of concern for manny research (e.g. [1], [13] and [14]). Possible damage (see [1]) during a space mission are altered cognitive function, including detriments in short-term memory, reduced motor function, and behavioral changes, which may affect performance and human health. This radiation induces (see [13]) changes in synaptic plasticity underlie many neuro degenerative conditions that correlate to specific structural alterations in neurons that are believed to be morphologic determinants of learning and memory. The authors in [14] exposed some rodents in separate groups to $5 \mathrm{cGy}^{48} \mathrm{Ti}$ or ${ }^{16} \mathrm{O}$ or $30 \mathrm{cGy}{ }^{16} \mathrm{O}$ during 12 and 24 weeks. Irradiation caused significant reductions in dendrite complexity, disrupted synaptic integrity and increased neurons inflammation. This cosmic radiation causes some cognitive dysfunction like in the novel object recognition (NOR) task, the object in place (OiP) task or the temporal order (TO) task (see [12], [13], [14]), .

Our topological data analysis will focus on the results of [14], however it can be extended to other ones. For 12 weeks, some mouses were exposed to cosmic radiation in the form of Titanium and Oxygen isotope, especially 5 or $30 \mathrm{cGy}^{48} \mathrm{Ti}$ or ${ }^{16} \mathrm{O}$ or $30 \mathrm{cGy}^{16} \mathrm{O}$. We choose to restrict our analysis to the results given by the Titanium irradiation, because that the damage caused by the Titanium irradiation is more important than any other cosmic radiation (see the figures here below from [14]). One other reason, to defend our choice, is that the Titanium isotope is the only one we can find in space.

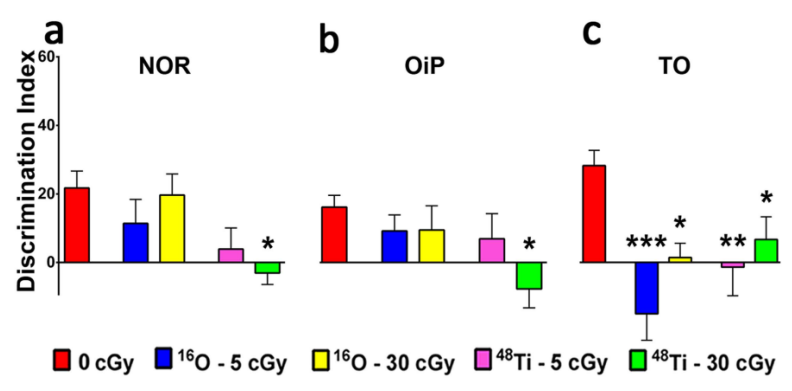

Figure 8: Cognitive deficits evaluated 12 weeks after cosmic radiation exposure.

Hence, this figure shows that a $30 \mathrm{cGy}^{48} \mathrm{Ti}$ particle irradiation significantly reduces the recognition memory, that it reduces the preference to explore an object found in a novel location and that it significantly impairs the memory by a reduced preference for the less recently explored object in the Temporal Order task (TO).
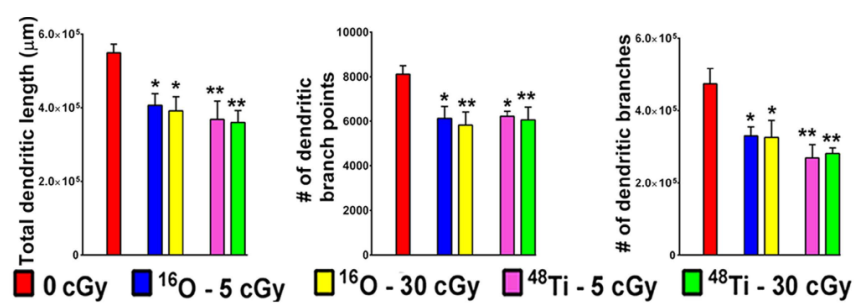

Figure 9: Reduced dendrite complexity of neurons after 12 weeks of cosmic radiation.

This quantification of the dendrite parameters, as bar charts, shows that the dendrite branching and length are significantly reduced 12 weeks after exposure to 5 or $30 \mathrm{cGy}^{48} \mathrm{Ti}$ or ${ }^{16} \mathrm{O}$ or $30 \mathrm{cGy}{ }^{16} \mathrm{O}$ particles.

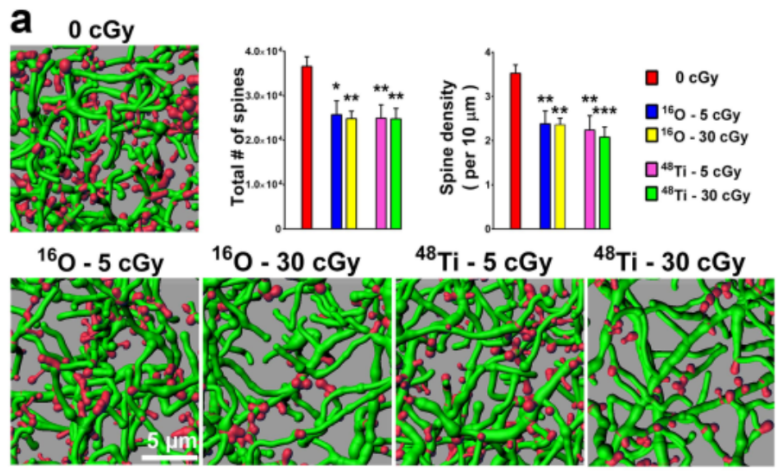

Figure 10: Reduced spine density of neurons after 12 weeks of cosmic radiation, see [12], [13], [14]

This representative digital images of 3D reconstructed dendrite segments (green) containing spines (red) in unirradiated (top left panel) and irradiated (bottom panels) brains, show that total spine numbers (left bar chart) and spine density (right bar chart) are significantly reduced after exposure to 5 or $30 \mathrm{cGy}^{48} \mathrm{Ti}$ or ${ }^{16} \mathrm{O}$ or $30 \mathrm{cGy}{ }^{16} \mathrm{O}$ particles.

Our approach is the following : We use this images to regionalize dendrites and spines and get, by using the Voronoi diagrams approach, some adjacent areas where spines and dendrites are concentrated. Once, the centers of the adjacent areas are connected, we get a Delaunay diagram which yields to a Rips complex on which TDA theory can be applied to get barecodes.

Firstly, to get the complex of Rips, we choose a threshold distance between the dendrites and spines region, that is the average distance between these regions for a graph exposed to 0cGy. With this threshold distance, we get a filtered graph which leads to a boundary matrix. 
Secondly, we implement a matrix reduction algorithm to pairs the persistent data, and represent These pairs represent the focus of cosmic radiation exposure. Finally, we calculate the diameter between two distant areas and compare the that obtained for another exposure to get prediction tool of the different cosmic radiations from the different obtained diameters of two focus of dendrites-spines cliques). The figure here below describes the pipe line of our approach.

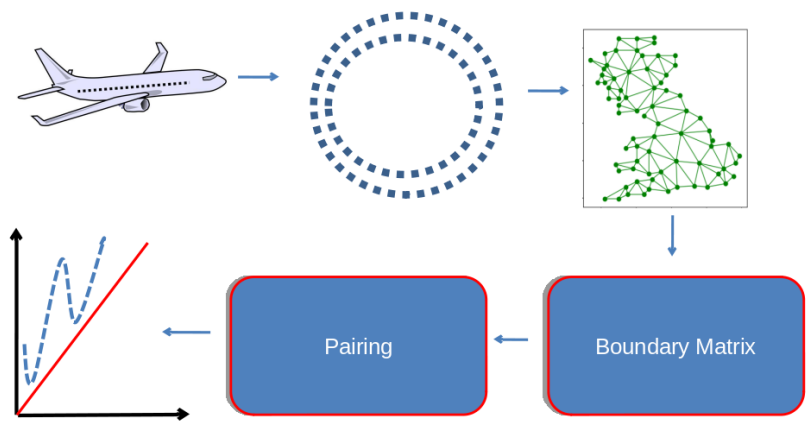

Figure 11: The pipe line to get persistent diagrams from digital images of a cosmic radiation.

In what follows, we more explain our approach step by step :

Step 1 : Get the adjacency matrix from an image (as explained by the image here below).

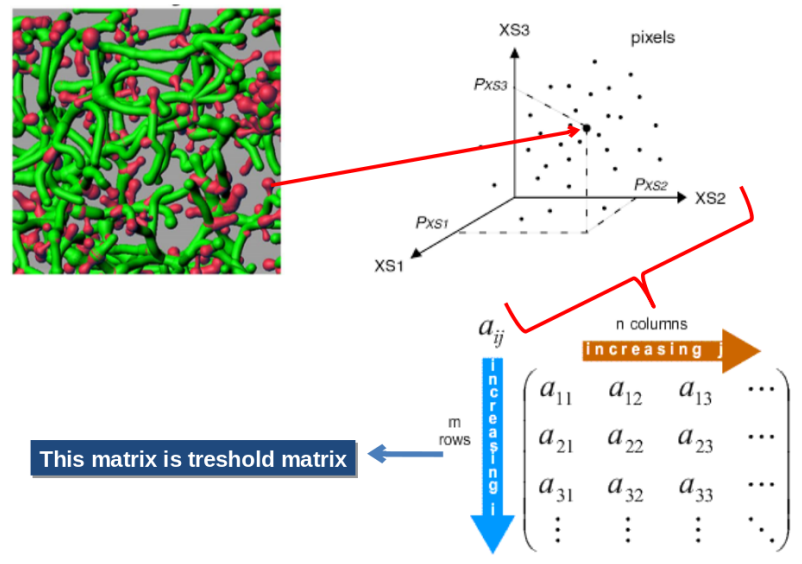

For this purpose we implemented the following algorithm :

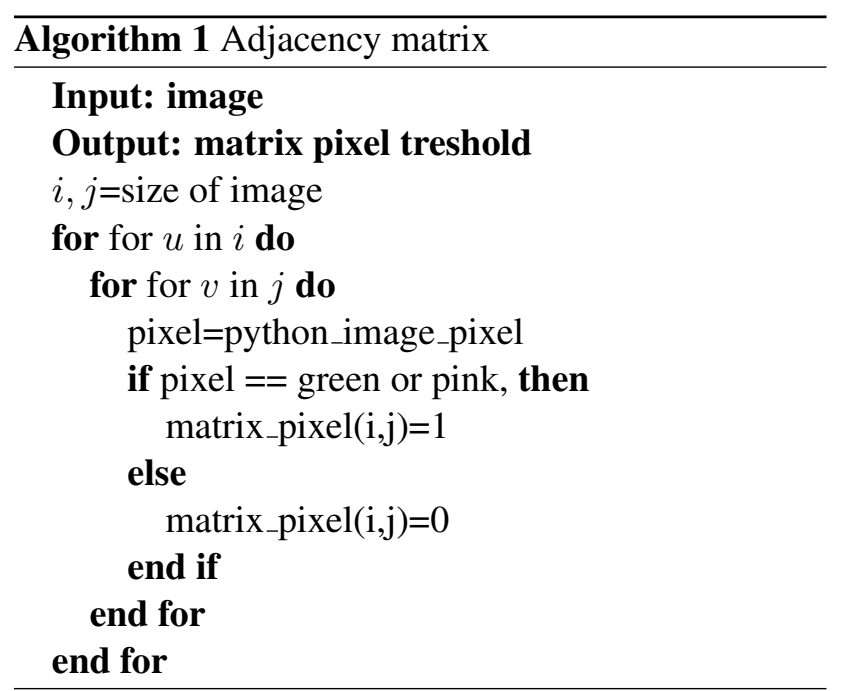

Step 2 : It consists to create the associated Voronoi diagrams by inserting the sites events in a file

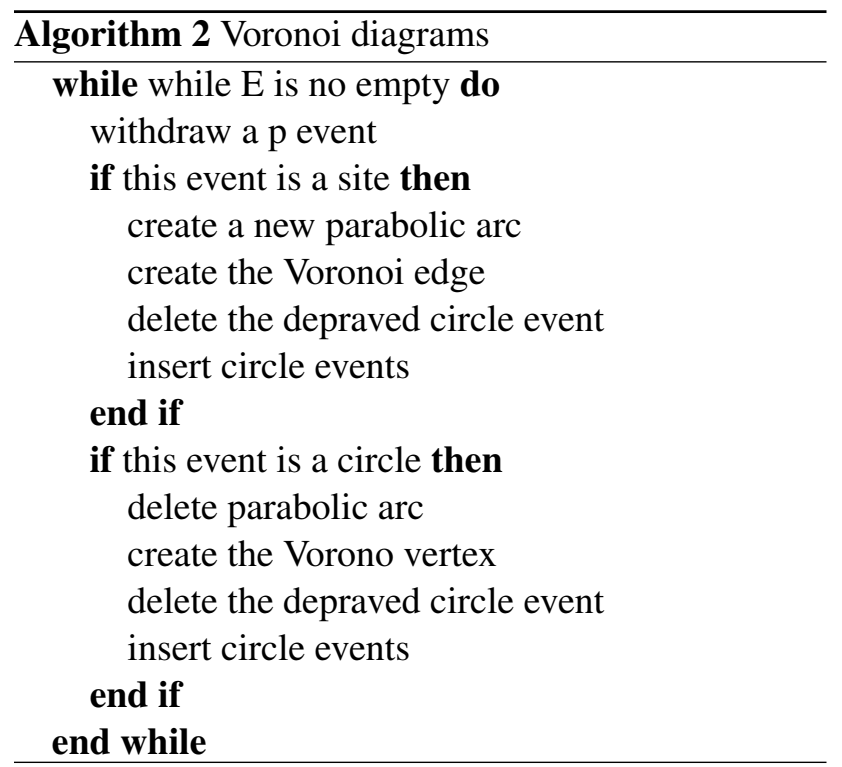

Here above some images of the dendrite and spines repartition after a cosmic radiation and their associated Voronoi diagrams.
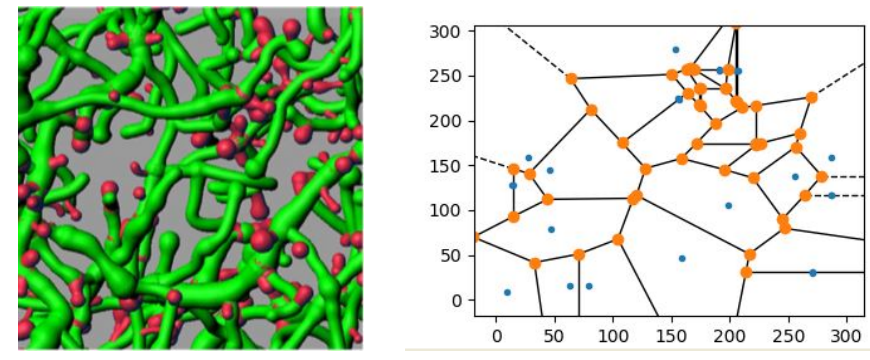

Figure 12: Voronoi diagram issued from a $5 \mathrm{cGy}^{48} \mathrm{Ti}$ radiation. 

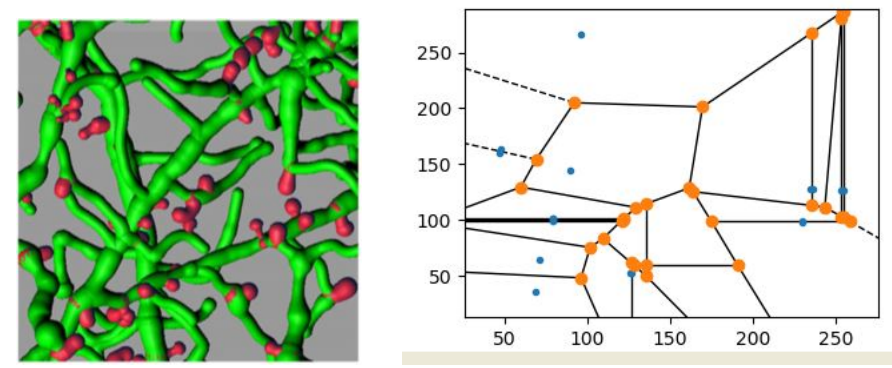

Figure 13: Voronoi diagram issued from a $30 \mathrm{cGy}^{48} \mathrm{Ti}$ radiation.

Step 3 : Build a Deluanay complex from the recently constructed Voronoi diagram as outlined by the following picture :
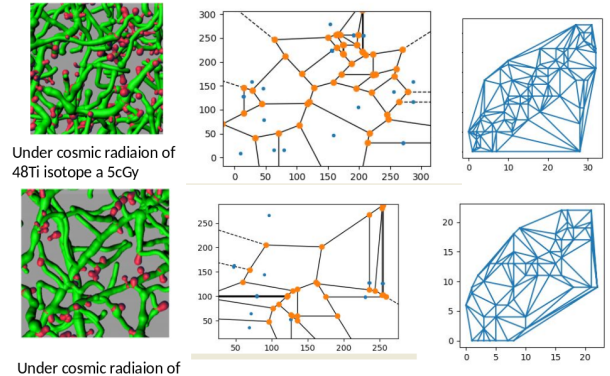

Under cosmic radiaion of
$48 \mathrm{Ti}$ isotope a $30 \mathrm{cGY}$
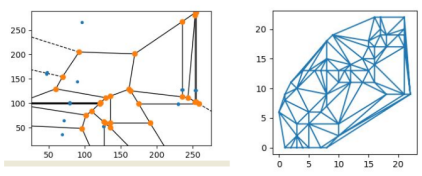

Figure 14: Examples of Delaunay complexes issued from Voronoi diagrams by using the Algorithm 3

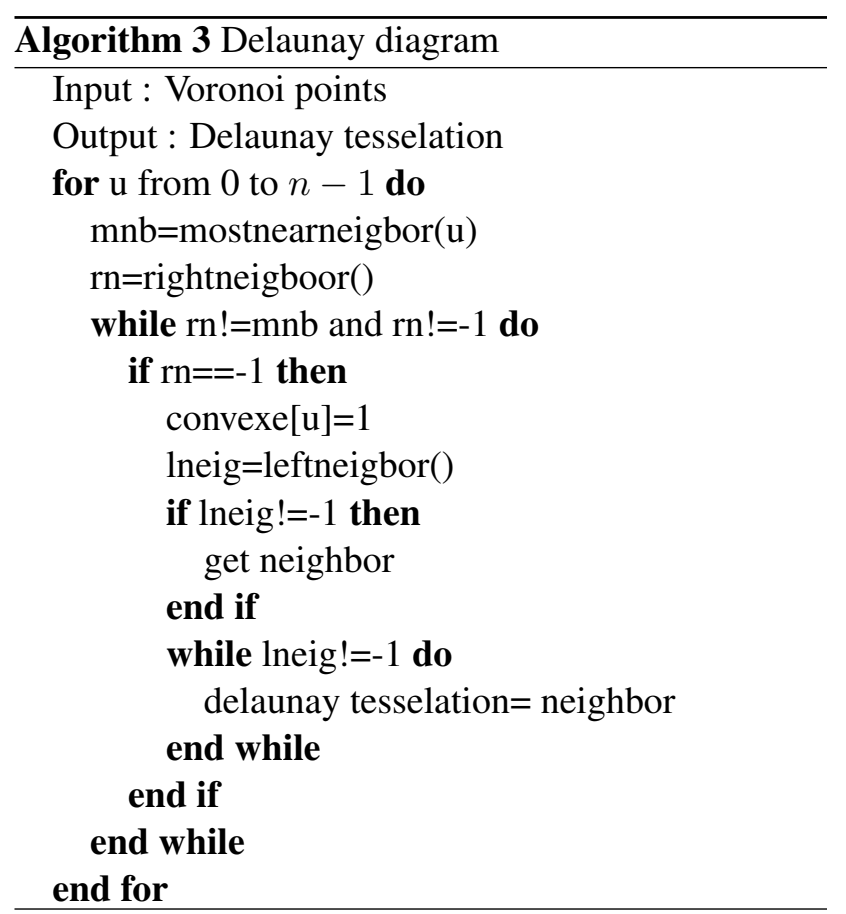

Step 4 : Its aim is to build a Rips complex from the obtained Delaunay triangulation. For this purpose we consider the Wasserstein distance in the following algorithm :

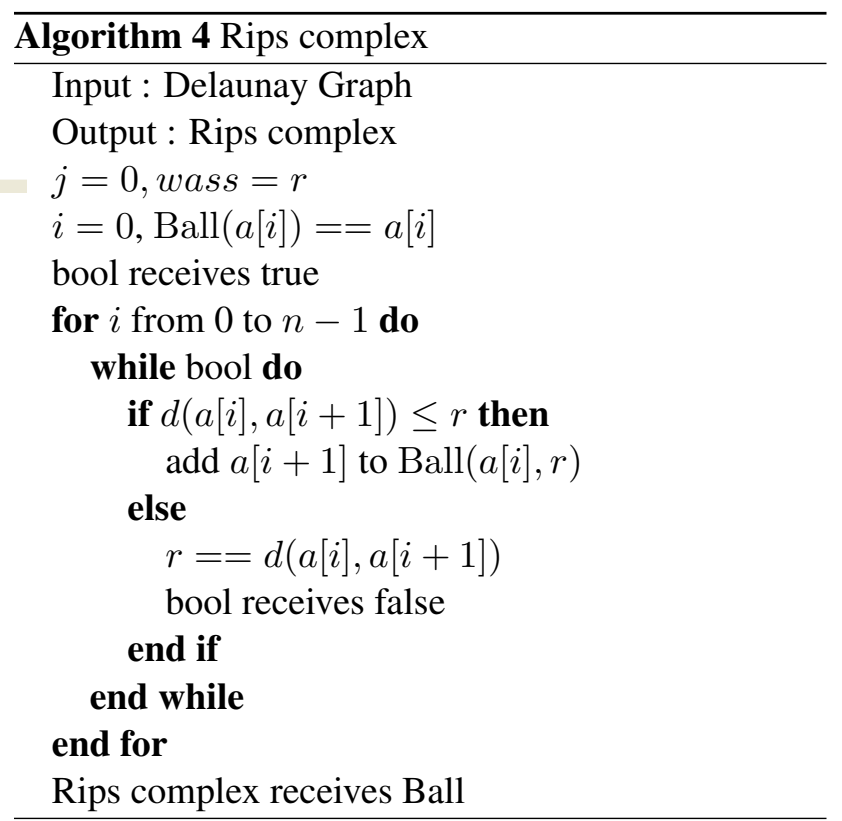

Step 5: The final step is to get the boundary matrix. The idea is that since for all $r_{0} \geq r$, we have $\operatorname{Rips}(N, r) \subset \operatorname{Rips}\left(N, r_{0}\right)$, then $\mathcal{F}=$ $\{\operatorname{Rips}(N, r), 0 \leq r \leq \operatorname{rmax}\}$ is a filtration. This Rips filtration induces a boundary matrix as stated by the following algorithm :

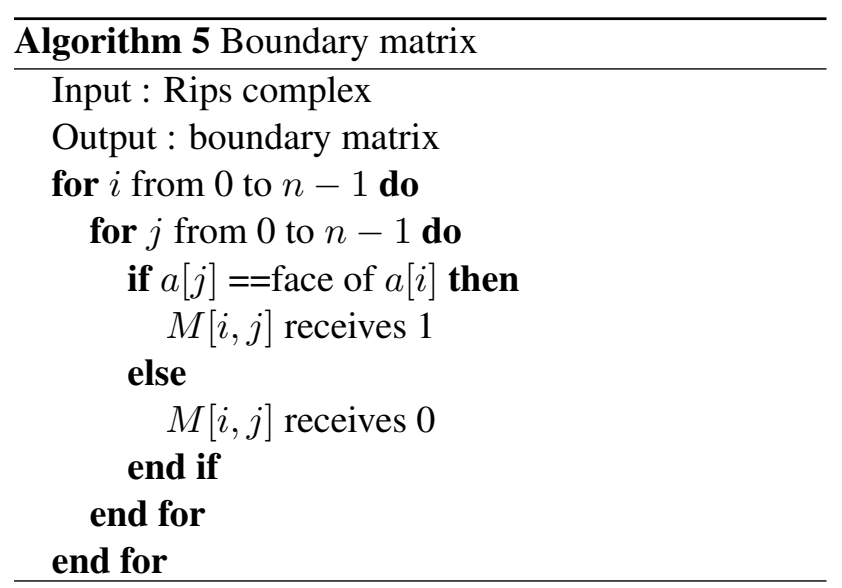

Finally to pair birth and death of cycles as mentioned in the subsection 3.2, we index columns by $j$, and $i=\ell(j)$ denotes the line that contains the lowest one in the column $j$, denoted $C_{j}$. A matrix is called reduced, when no two different columns have their lower at the same level. To get a reduced boundary matrix, we appeal this algorithm: 


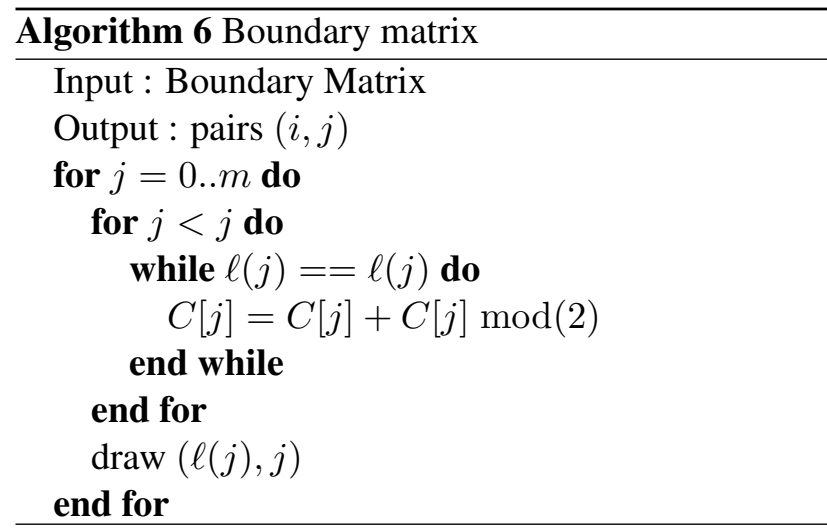

The fruit of this implementation is the following persistent diagrams

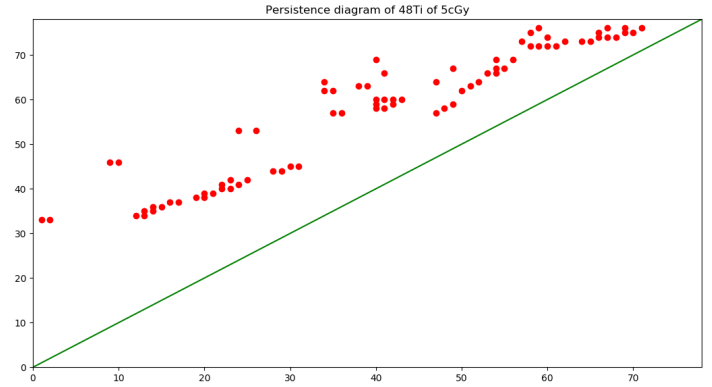

Figure 15: Persistent diagram that represents the dendrite and spines density under $5 \mathrm{cGy}^{48} \mathrm{Ti}$ radiation.

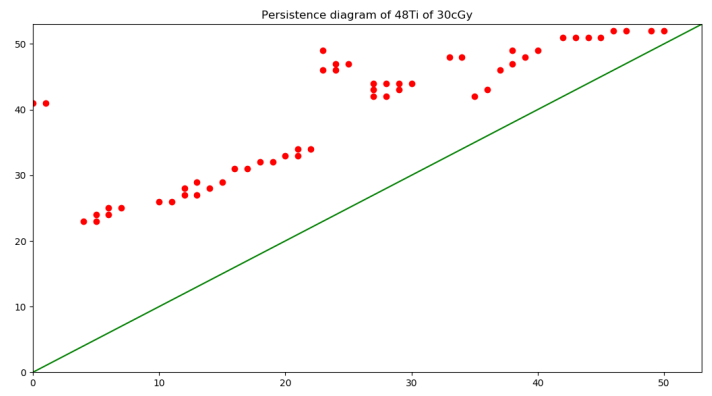

Figure 16: Persistent diagram that represents the dendrite and spines density under $30 \mathrm{cGy}^{48} \mathrm{Ti}$ radiation.

\section{Conclusion}

Our persistent diagrams show clearly how much the $30 \mathrm{cGy}^{48} \mathrm{Ti}$ radiations damage deeply neuronal tissue more than the $5 \mathrm{cGy}^{48} \mathrm{Ti}$ radiations. This shows the powerful of the topology approach in the data analysis. Indeed, the experiences leaded by the the Limoli staff (as outlined here below) were unable to differentiate the negative effects on the neuronal tissue after radiation $5 \mathrm{cGy}^{48} \mathrm{~T}$ and $30 \mathrm{cGy}^{48} \mathrm{~T}$.

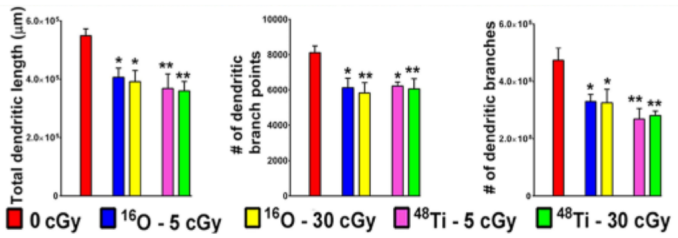

Figure 17: Dendrite density under different radiations

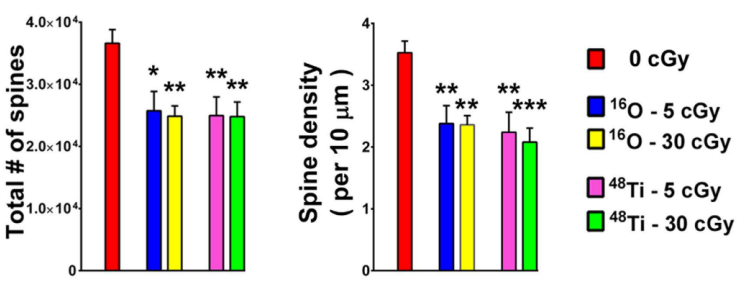

Figure 18: Spines density under different radiations

\section{Conclusion}

The cosmic ray is classical scientific subject, in the past many results have been obtained in laboratories, or by some empiric other ones. We propose an algorithm that will explore the data from images and get deep results by using persistent homology. For our knowledge, this is the first persistent homology approach to identify the intensity of cosmic ray of one isotop by regarding his damage throughout the density of spine.

The impact in neuroscience of our algorithm, of complexity $2 n \ln (n)$, is to get a ready tool that can identify the intensity and type of isotop in a cosmic radiation, it is fast recognition process of pathology related to cosmic ray. In fact, since our algorithm encode the intensity of a cosmic ray into a barre code, the further application we work on is to develop a software to identify cosmic radiation by analysis some brain images to localise damaged spines and dendrites damaged regions: the output will be barcodes which encode this information.

The reader can see how mathematical abstractions seem so often to be so powerfully predictive in the real world, and how pure mathematics (topology) and applied one (algorithmic) can be married in persistent homology to get kind of applications in real life.

An unavoidable question that doctors and patients with brain tumours could not ignore any longer is: What really happens to your brain when you blast it with radiation? They found that their once-bushy neurons looked like they were curbside hedges after 
the township tree had gotten through them, they were emasculated from their branches and leaves. Based on this topological approach, we are working on implementing a software to predict from any IREM image of the neuronal tissue, the negative possible impact of addict use of the electronic devices.

\section{References:}

[1] F.A. Cucinotta, M. Alp, F. M. Sulzman and M. Wang. Space radiation risks to the central nervous system, Life Sciences in Space Research, Vol. 2 (2014) 54-69.

[2] G. Carlsson. Topology and data. Bull. AMS, vol 46 (2009), 255-308.

[3] G. Carlsson, A. Zomorodian, A. Collins, and L. Guibas. Persistence barcodes for shapes, International Journal of Shape Modeling, vol. 11 (2005), 149-187.

[4] H. Edelsbrunner, D. Letscher, and A. Zomorodian. Topological persistence and simplification. Discrete Comput. Geom., vol 28 (2002), 511-533.

[5] H. Edelsbrunner, J. Harer. Persistent homology : a survey. Contemporary mathematics, 453 (2008), 257-282.

[6] H. Edelsbrunner, J. Harer. Computational Topology : An introduction. American Mathematical Society, Providence (2010).

[7] E. Fermi, On the origin of cosmic radiation. Phys. Rev. 75 (1949), 1169-1174.

[8] A. Hatcher. Algebraic topology, Cambridge University Press (2002).

[9] J. R. Munkres. Elements of Algebraic Topology, Addison-Wesley, Redwood City, California (1984).

[10] Obayashi, Y. Hiraoka, and M. Kimura. Persistence diagrams with linear machine learning models. Journal of Applied and Computational Topology, 1(3-4):421-449, 2018.

[11] N. Otter, M. A. Porter, U. Tillmann, P. Grindrod, and $\mathrm{H}$. A. Harrington. A roadmap for the computation of persistent homology, EPJ Data Science, vol. 6, num. 17 (2017), 1-38.

[12] V.K Parihar, B. D. Allen, K. K. Tran, T. G. Macaraeg, E. M. Chu, S. F. Kwok, N. N. Chmielewski, B. M. Craver, J. E. Baulch, M. M. Acharya, F. A. Cucinotta and Ch. L. Limoli. What happens to your brain on the way to Mars, Life Sciences in Space Research. Vol. 1, no. 4 (2015), $1-6$.

[13] V. K. Parihar and Ch. L. Limoli. Cranial irradiation compromises neuronal architecture in the hippocampus. PNAS, Vol. 110 , no 31 (2013), 12822-12827.

[14] V. K. Parihar , B. D. Allen, Ch. Caressi, S Kwok, E. Chu, K. K. Tran, N. N. Chmielewski, E. Giedzinski, M. M. Acharya, R. A. Britten, J. E. Baulch and Ch. L. Limoli. Cosmic radiation exposure and persistent cognitive dysfunction, Scientific Reports, Vol. 6 (2016), 34774-34788.

[15] R. D. Richtmyer and E. Teller, On the origin of cosmic rays. Phys. Rev. 75 (1949), 1729-1735.

[16] Yanning Zhou, Omer Fahri Onder, Qi Dou, EfstratiosTsougenis, Hao Chen, and Pheng-Ann Heng. CIA-Net: Ro-bust nuclei instance segmentation with contour-aware infor-mation aggregation. In International Conference on Information Processing in Medical Imaging, 2019.

[17] A. Zomorodian and G. Carlsson. Computing persistent homology, Discrete and Computational Geometry, vol. 33, num. 2 (2005), 247-274. 